Check for updates

Cite this: RSC Adv., 2017, 7, 34226

Received 26th April 2017

Accepted 3rd July 2017

DOI: $10.1039 / c 7 r a 04709 f$

rsc.li/rsc-advances

\section{Ethylenediamine functionalized carbon nanoparticles: synthesis, characterization, and evaluation for cadmium removal from water}

\begin{abstract}
Luthando Tshwenya $^{\mathrm{ab}}$ and Omotayo A. Arotiba (iD *abc
This work illustrates the functionalization of glucose-derived carbon nanoparticles (CNPs) with ethylenediamine and their possible application in the removal of cadmium from water. A fast microwave assisted method was used to synthesise the CNPs resulting in good yields and an abundance of functional groups (carboxyl, hydroxyl groups etc.) which served as anchor sites for modification and adsorption. The pristine glucose-derived carbon nanoparticles (GCNPs) and functionalized CNPs (EDAGCNPs) were characterised using TEM, BET, XRD, FTIR, surface charge and elemental analysis. Batch adsorption experiments were used to study the effect of factors such as $\mathrm{pH}$, adsorbent dosage and the initial concentration of the metal ion of interest. From the adsorption studies, kinetics and sorption equilibrium were also studied, implying that both carbon nanoparticles could effectively remove cadmium through chemisorption (the data fitting a pseudo-second-order model), with maximum sorption capacities of 10.314 and $18.708 \mathrm{mg} \mathrm{g}^{-1}$ for GCNPs and EDA-GCNPs, respectively.
\end{abstract}

\section{Introduction}

The release of highly toxic metals often regarded as "heavy" into surface and ground waters due to acid mine drainage (AMD), industrialisation and other activities has been known to cause severe environmental and health related problems. These metals are particularly of a major concern due to their tendency to be non-biodegradable in nature and leading to long-term accumulation in the ecosystem including humans. ${ }^{1-3}$ Cadmium (Cd) a by-product of zinc mining is an example of such toxic metal ions. Once in the human system, cadmium is known to build up primarily in the kidneys, throughout life without being excreted, ${ }^{4}$ ultimately damaging the renal, digestive and even the nervous systems. ${ }^{5}$ Thus the maximum tolerable cadmium limit in drinking water is $3 \mu \mathrm{g} \mathrm{L}^{-1}$ as reported by the World Health Organization (WHO).

Various remediation technologies have been developed and reported for the removal of cadmium and other heavy/toxic metals. These methods include chemical precipitation, ${ }^{6}$ membrane filtration, ${ }^{7}$ ion-exchange ${ }^{8}$ electro-coagulation, ${ }^{9}$ and adsorption. ${ }^{\mathbf{1 0 - 1 3}}$ Each of these methods has its merits and limitations. For example, chemical precipitation involves the use of expensive materials and often generates a secondary toxic waste, membranes are subjected to fouling rendering them

${ }^{a}$ Department of Applied Chemistry, University of Johannesburg, Doornfontein, South Africa.E-mail: oarotiba@uj.ac.za

${ }^{b}$ DST/Mintek Nanotechnology Innovation Centre, University of Johannesburg, South Africa

${ }^{c}$ Centre for Nanomaterials Science Research, University of Johannesburg, South Africa ineffective after a few times of usage and the rest are just inapplicable for commercial scale.

When water treatment techniques are compared according to their cost-effectiveness, efficiency, ease of operation and availability based on a wide range of materials (both natural and man-made), adsorption is found to be among the top techniques that meet these criteria. Adsorption has been used for the removal of different pollutants (both inorganic and organic), with cadmium included [ref]. Some other advantages of adsorption include ease of waste handling after treatment, reusability, use of easily accessible or low-cost materials (e.g. activated carbon), portability of facilities and much more. One of the limitations of adsorption, however, is its limited commercial application. This limitation can be due to some factors such as cost, inability to adsorb toxic metals at low concentration and lack of robustness towards the removal of wider range of pollutants per adsorbent. ${ }^{\mathbf{1 4}}$ Carbon nanomaterials have been highly researched recently owing to their unique properties and wide applicability. However, the production of these materials mostly requires stringent conditions and expensive synthesis tools. Among the different carbon nanomaterials, carbon nanoparticles (CNPs) are slowly attracting attention owing to their ease of synthesis, fluorescent nature, relatively low/non-toxicity, high surface area and functionalizability. ${ }^{15-17}$ These materials are referred to as carbon nanoparticles because they consist primarily of carbon in the form of nearly spherical particles that are between 10 and $100 \mathrm{~nm}$ in size. ${ }^{\mathbf{1 8 , 1 9}}$ Additionally, they usually contain oxygen and hydrogen which makes them hydrophilic, and dispersible in aqueous solutions. ${ }^{17}$ 
The modification of CNPs (and other nanocarbons) either by physical or chemical treatments, is known to induce new properties or enhance some already existing properties. ${ }^{19,20}$ Surface modification is best needed to break the strong cohesive forces that cause aggregation, thus promoting dispersibility and stability of the particles. ${ }^{21}$ Functionalization of amide-amine type is considered as one of the best methods to introduce these desirable properties, ${ }^{22}$ making CNPs ideal for various applications including catalysis, and medicine. ${ }^{23,24}$ Moreover, adding functional groups such as hydroxyl, carboxylate, sulphate and amino groups on surfaces (including carbons) is known to increase their heavy metal affinity (through chelation and complexation), making them better adsorbents and more desirable for water treatment. ${ }^{25,26}$

Reports on the use of CNPs as adsorbents are scanty with those tailored towards heavy metal removal are even less uncommon in the literature. A study conducted by Ruparelia et al. reported that carbon nanoparticles such as soot had a potential of removing harmful heavy metals from water. ${ }^{27}$ However, the method involved in preparing the reported CNPs was tedious and time-consuming. Thus studies on alternative synthesis routes (that are low cost and present good yields) and functionalization of CNPs will be plausible so as to widen the applicability of this relatively new nanocarbon. Herein, CNPs were synthesised via a microwave-assisted carbonisation process from the dehydration of glucose. This approach is very rapid and gives good yields in contrast to the high-cost and energy consuming methods typically used to synthesise most carbon materials. The CNPs prepared were functionalized with ethylenediamine (EDA) and used for the first time in $\mathrm{Cd}^{2+}$ removal (adsorption) from the water. The choice of EDA was motivated by its nitrogen groups that have lone pairs which can chelate with most metal ions.

\section{Experimental}

\subsection{Chemicals}

Chemicals such as $\mathrm{D}(+)$ glucose anhydrous (platinum line, AR), orthophosphoric acid (85\%, platinum line), ethanol absolute (96\%, ACS reagent) were obtained from Associated Chemical Enterprise (South Africa). Ethylenediamine ( $\geq 99 \%$, ReagentPlus), HATU $(97 \%, 445460), \mathrm{Cd}\left(\mathrm{NO}_{3}\right)_{2} \cdot 4 \mathrm{H}_{2} \mathrm{O}(99.997 \%$, trace metals basis), $\mathrm{HNO}_{3}\left(99.999 \%\right.$, trace metals basis) and $\mathrm{NH}_{4} \mathrm{OH}$ (28-30\%, ACS reagent) were purchased from Sigma-Aldrich (South Africa).

\subsection{Preparation of carbon nanoparticles}

Carbon nanoparticles (CNPs) were synthesised by slightly modifying a synthesis method previously reported by Chandra and co-workers. ${ }^{28}$ This was achieved by dissolving $2 \mathrm{~g}$ of glucose in $10 \mathrm{~mL}$ of water in a $500 \mathrm{~mL}$ beaker. To the dissolved solution, a $20 \mathrm{~mL}$ volume of orthophosphoric acid was added followed by mixing. The beaker with the mixed contents was then transferred into a microwave $(100 \mathrm{~W})$ for 5 minutes, then left aside to cool, after which, $100 \mathrm{~mL}$ of water was added, followed by mixing and sonication to further disperse the nanoparticles. To isolate the CNPs from the solution, the mixture was centrifuged at $4000 \mathrm{rpm}$ for $10 \mathrm{~min}$. The precipitate was washed several times with deionized water until an almost neutral $\mathrm{pH}$ was obtained from the filtrate. Finally, the sample was filtered then dried at $40{ }^{\circ} \mathrm{C}$ using a vacuum oven. The synthesis of CNPs was carried out using an LG microwave operated at $100 \mathrm{~W}$. A yield of $1.29 \mathrm{~g}, 64.5 \%(\mathrm{~m} / \mathrm{m})$ was obtained.

\subsection{Preparation of ethylenediamine functionalized CNPs}

A $200 \mathrm{mg}$ mass of the previously synthesised CNPs was dispersed in $100 \mathrm{~mL}$ DMF by sonication, then stirred at $30{ }^{\circ} \mathrm{C}$ for an hour. To the dispersed solution, $100 \mathrm{~mL}$ of ethylenediamine (EDA) was added followed by stirring for another hour. Afterwards, $10 \mathrm{mg}$ of the coupling agent (HATU) was added, and the mixture was refluxed at $60{ }^{\circ} \mathrm{C}$ for $6 \mathrm{~h}$ under stirring to carry out the amidation step. The product was diluted with $500 \mathrm{~mL}$ ethanol, filtered, then washed a couple of times with ethanol. The final product was then dried at $50{ }^{\circ} \mathrm{C}$ for $12 \mathrm{~h}$, yielding $209.3 \mathrm{mg}, 104.65 \%(\mathrm{~m} / \mathrm{m})$.

\subsection{Characterizations}

2.4.1. TEM analysis. A small portion of the solid sample was dispersed in ethanol using an ultrasonicator, then a single drop of the dispersed solution was dropped onto a carboncoated copper grid, which was then inserted onto a JEM 2100 transmission electron microscope (JEOL, Japan), operated at a voltage of $200 \mathrm{kV}$, to determine the morphology and the size of the particles.

2.4.2. FT-IR analysis. FT-IR spectra were recorded on a Perkin-Elmer Spectrum 100 spectrometer (Perkin-Elmer, USA) at room temperature by the $\mathrm{KBr}$ pellet technique, in the region $400-4000 \mathrm{~cm}^{-1}$.

2.4.3. Elemental composition analysis. A Leco CHNS analyser was used to obtain the carbon, hydrogen, nitrogen and sulphur composition of the samples (LECO, USA).

2.4.4. Point of zero charge $\left(\mathbf{p H}_{\mathbf{p z c}}\right)$ analysis. The surface charge/point of zero charge analysis for the adsorbents at hand was carried out using a Nano-ZS Zetasizer (Malvern, UK).

2.4.5. Surface area and pore volume analysis. The specific surface area and porosity studies were performed by nitrogen adsorption and desorption isotherms (BET method) determined at $77 \mathrm{~K}$ using an ASAP2020 surface area and porosity analyser (Micromeritics Instrument, USA). Prior to the analysis, each sample was degassed at $100{ }^{\circ} \mathrm{C}$ for 3 hours using nitrogen gas.

2.4.6. XRD analysis. XRD measurements were carried out using a powder Rigaku miniflex 600 diffractometer, with a $\mathrm{Cu} \mathrm{K} \alpha$ radiation source (wavelength, $\lambda=0.1542 \mathrm{~nm}$ ) operated at $40 \mathrm{kV}$ and $30 \mathrm{~mA}$ for $2 \theta$ values from $10^{\circ}-90^{\circ}$ (Rigaku, Japan).

\subsection{Batch adsorption experiments}

A standard solution of $1000 \mathrm{mg} \mathrm{L}^{-1} \mathrm{Cd}(\mathrm{II})$ was prepared by dissolving cadmium nitrate tetrahydrate in a $1 \% \mathrm{HNO}_{3}$ solution (for standard preservation), from which all other experimental 
solutions of the required $\mathrm{mg} \mathrm{L}^{-1}$ concentrations were prepared by dilution with MilliQ water. Factors such as $\mathrm{pH}$, temperature, contact time, and the initial concentrations of the $\mathrm{Cd}^{2+}$ solution were investigated, in order to understand the $\mathrm{Cd}(\mathrm{II}) /$ adsorbent interaction better. A temperature controlled water bath set at a constant shaking speed (200 rpm) was used in all batch experiments conducted. The effect of $\mathrm{pH}$ on the removal of cadmium was studied over a $\mathrm{pH}$ ranging 3-8 using a known volume of $\mathrm{Cd}(\mathrm{II})\left(10 \mathrm{mg} \mathrm{L}^{-1}\right)$. The $\mathrm{pH}$ adjustments were done using a portable digital $\mathrm{pH}$ meter (Ohaus, USA) by adding $0.1 \mathrm{M}$ $\mathrm{HNO}_{3}$ and $0.1 \mathrm{M} \mathrm{NH}_{4} \mathrm{OH}$. To study the effect of adsorbent dosage a specified dose (ranging from 5-100 mg) was added to constant volumes of $\mathrm{Cd}$ (II) solutions, agitated at $200 \mathrm{rpm}$ at $25{ }^{\circ} \mathrm{C}$ for $120 \mathrm{~min}$. The effect of contact time and the kinetics were investigated by agitating and withdrawing aliquots at an interval of 5 to $210 \mathrm{~min}$ to determine metal ion concentration, whilst all other parameters are constant. Similarly, adsorption isotherm studies were carried out by varying $\mathrm{Cd}(\mathrm{II})$ ion concentration from 5 to $50 \mathrm{mg} \mathrm{L}^{-1}$ at an optimum $\mathrm{pH}$ of 6 . Finally, thermodynamics studies were conducted by varying the temperature from $20-35{ }^{\circ} \mathrm{C}(293-308 \mathrm{~K})$, whilst all other parameters were fixed. All reaction mixtures after adsorption were passed through a $0.22 \mu \mathrm{m}$ PVDF filter, and the supernatants analysed for residual $\mathrm{Cd}(\mathrm{II})$ ions concentration using an ICP-OES.

To ensure accuracy and reliability of the data collected, blank tests were conducted, these tests were performed by adding the adsorbate $\left(\mathrm{Cd}^{2+}\right)$ solution onto a plastic container, and subjecting it to agitation for a specific time at a specific temperature, without the adsorbent present (the same way all other batch adsorption experiments were conducted). The fact that there was no significant changes in $\mathrm{Cd}(\mathrm{II})$ concentration from those diluted from the standard/stock and the blanks prepared indicated that concentration changes observed in the real adsorption studies were due to adsorption by the adsorbents only and that the plastic vessel/container used in all experiments had negligible effect. All adsorption experiments were carried out in triplicate, and the results reported were averaged from the sets. A water bath shaker (207, Rochelle lab equipment, SA) was used for dispersion of the samples during batch adsorption experiments. The cadmium ion concentrations (before and after adsorption) were measured using an ARCOS ICP-OES, equipped with a Cetac ASX-520 auto-sampler (Spectro, USA).

2.5.1. Removal rate and removal capacity. The \%Cd(II) removed as well as the adsorption capacities of the adsorbents were calculated using the equations reported below;

$$
\begin{gathered}
\% \mathrm{Cd}(\mathrm{II}) \text { removed }=\frac{\left(C_{\mathrm{o}}-C_{\mathrm{f}}\right)}{C_{\mathrm{o}}} \times 100 \% \\
Q_{\mathrm{e}}=\frac{\left(C_{\mathrm{o}}-C_{\mathrm{f}}\right) V}{M}
\end{gathered}
$$

where $C_{\mathrm{o}}$ and $C_{\mathrm{f}}$ are the initial and final $\mathrm{Cd}^{2+}$ concentrations respectively in $\mathrm{mg} \mathrm{L}^{-1}$. $V$ is the volume of $\mathrm{Cd}$ (II) solution in litres (L) and $M$ is the mass of the adsorbent in grams (g).

\section{Results and discussion}

\subsection{Characterizations}

3.1.1. TEM analysis. Fig. 1a and b show TEM images of glucose-derived carbon nanoparticles (GCNPs) and the ethylenediamine modified carbon nanoparticles. From these images, a spherical shape is observed in both materials, as well as a higher degree of aggregation seen on GCNPs in comparison to the EDA-GCNPs. Such chain-like aggregations were also reported by Trostová et $a .^{21}$ and Chandra et $a .^{28}$ The decreased aggregation of the EDA-GCNPs is believed to be as a result of the sonication step as well as the incorporation of the amine groups which broke the cohesive forces which held the particles together during the functionalization step. From the TEM distribution plots (Fig. 1c and d), the average particle size of the GCNPs was found to be $38.01 \mathrm{~nm}$ and upon modification, with EDA the particle decreased to $29.78 \mathrm{~nm}$. This decrease in size is believed to be as a result of the introduction of ethylenediamine groups which has been previously reported to reduce agglomeration. Furthermore, the sonication step involved in this process might have further dispersed the particulates that were agglomerated resulting in smaller particles. There were no significant differences in the structures of the carbon nanoparticles before and after functionalization.

3.1.2. FT-IR analysis. FTIR spectroscopy (Fig. 2a) was used to verify the present functional groups before and after functionalization. The broad absorption peak at around $3350 \mathrm{~cm}^{-1}$ can be assigned to $\mathrm{O}-\mathrm{H}$ and $\mathrm{N}-\mathrm{H}$ stretching vibrations expected before and after functionalization, respectively. ${ }^{21,29}$ The fact that the bands at 2848 and $2917 \mathrm{~cm}^{-1}$ are greatly strengthened on the EDA-GCNPs spectrum serves as evidence for the introduction of methylene groups $(\mathrm{C}-\mathrm{H}$ bonds from ethylenediamine) onto the surface of the carbon nanoparticles. The $\mathrm{C}=\mathrm{O}$ stretching peak that originally appeared at $1710 \mathrm{~cm}^{-1}$ on the spectrum of the GCNPs, completely disappears or shifts to a lower position in the spectra of EDA-GCNPs, or coincides with the $\mathrm{C}=\mathrm{C}$ band at $1610 \mathrm{~cm}^{-1}$; whilst the $\mathrm{C}-\mathrm{O}$ (initially at 1019 $\mathrm{cm}^{-1}$ ) stretching shifts to $1087 \mathrm{~cm}^{-1}$ on the EDA-GCNPs, due to the $\mathrm{N}-\mathrm{H}$ stretching of amine groups. In the EDA-GCNPs spectrum distinct peaks appearing at 1476, 1370 and $1306 \mathrm{~cm}^{-1}$ can be attributed to the anti-symmetric $\mathrm{C}-\mathrm{N}$ stretching vibrations coupled with the out-of-plane $\mathrm{NH}_{2}$ and $\mathrm{NH}$ modes. ${ }^{30,31}$

3.1.3. Elemental composition analysis. A CHNS microanalyzer was used to determine the elemental composition of the glucose-derived carbon nanoparticles (GCNPs) as well as the composition after functionalization (EDA-GCNPs). The carbon, hydrogen and nitrogen content detected from this analysis are provided in Table 1 . The presence of nitrogen, as well as an increase in hydrogen content in the EDA-GCNPs, served as an indication of amine groups being introduced after the functionalization step. The oxygen content can be obtained by a mass difference supposing that the remaining element is oxygen. ${ }^{32}$ The decrease in oxygen content upon EDA functionalization can suggest that most of the carboxylic groups that were present on the GCNPs surface were converted to amide groups indicating a successful grafting. 
a)

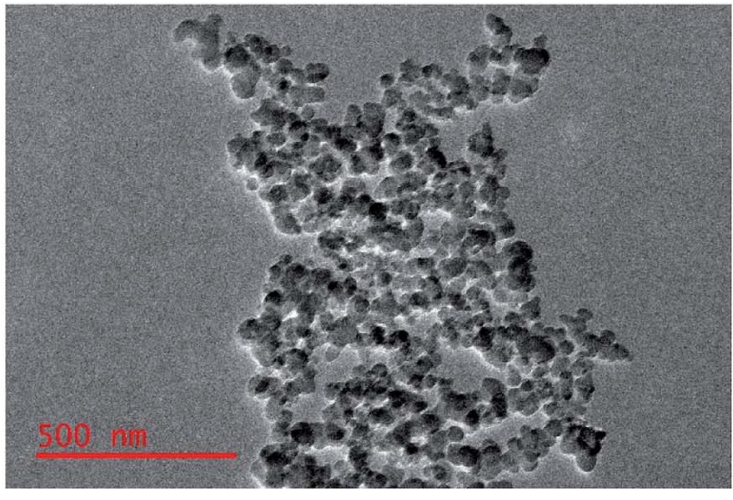

b)
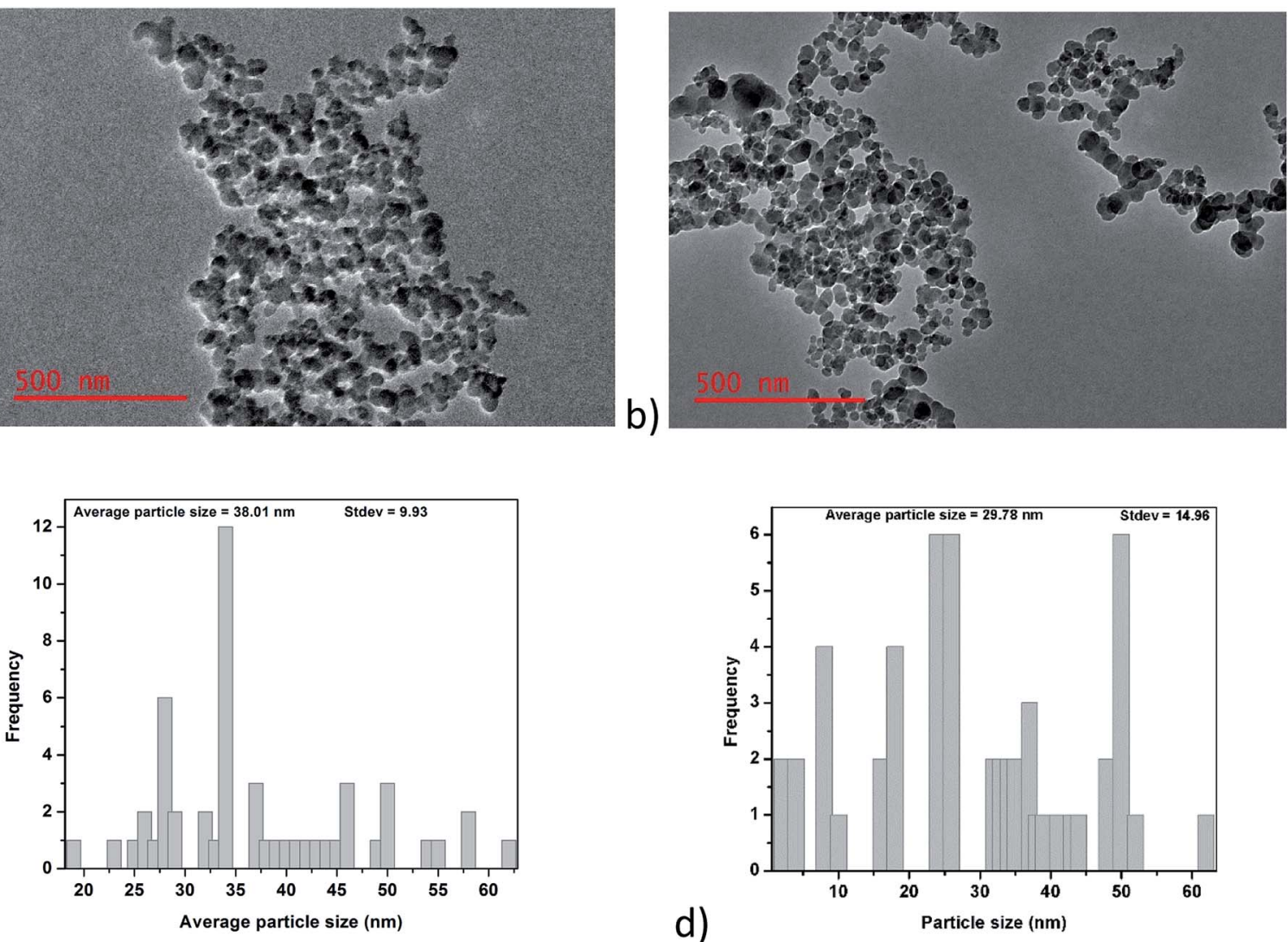

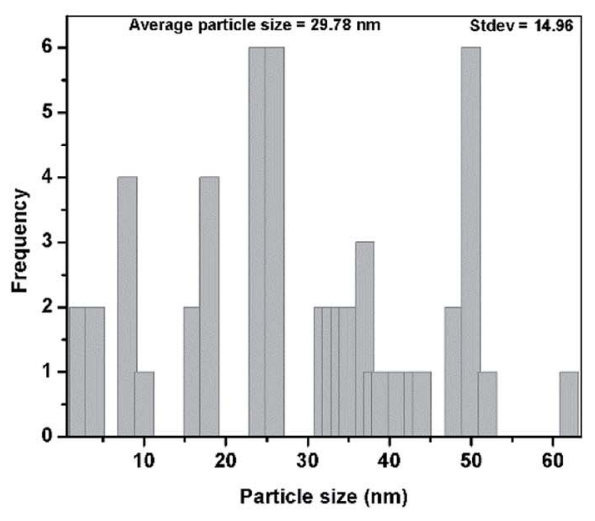

Fig. 1 Shows; (a) and (b) TEM images, as well as (c) and (d) distribution curves for GCNPs and EDA-GCNPs, respectively.

3.1.4. Point of zero charge analysis. Zeta potential studies (Fig. 2b) were conducted from pH 2-12, and the point of zero charges $\left(\mathrm{pH}_{\mathrm{PZC}}\right)$ of the two materials was recorded as 2.4 and 3.5 for the GCNPs and EDA-GCNPs, respectively. This, therefore, meant that below these $\mathrm{pH}$ values, the adsorbent's surface is positively charged, favouring anionic adsorption. Above the recorded $\mathrm{pH}_{\mathrm{PCZs}}$ the materials exhibit a negative surface charge, favouring cationic adsorption via an electrostatic interaction between the negative surface (from carboxyl/amine groups present) of the adsorbent and the positive metal ion. This, therefore, meant that these materials showed potential as adsorbents for metal removal, and particularly positively charged metals, as long as $\mathrm{pH}$ was above 3.5 . Thus this electrostatic attraction was exploited for metal ion adsorption in this research.

3.1.5. Surface area and pore volume analysis. From the adsorption isotherms (Fig. 3a and b), it is clearly seen that both the synthesised materials exhibit a type (IV) nature. Characteristic features of the type IV isotherm are its hysteresis loop, which is associated with capillary condensation taking place in

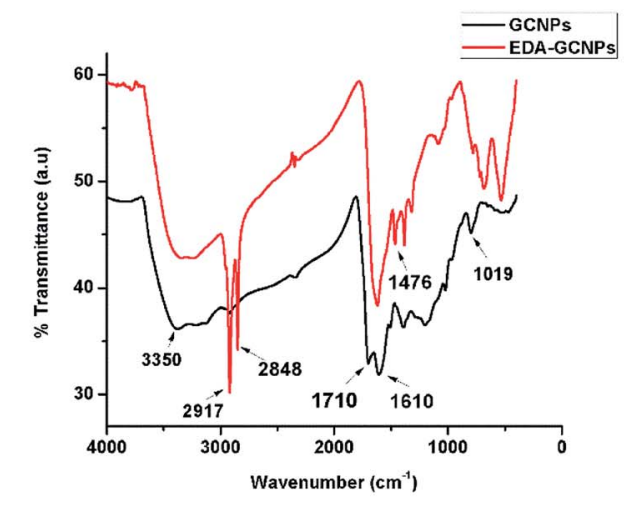

a)

b)

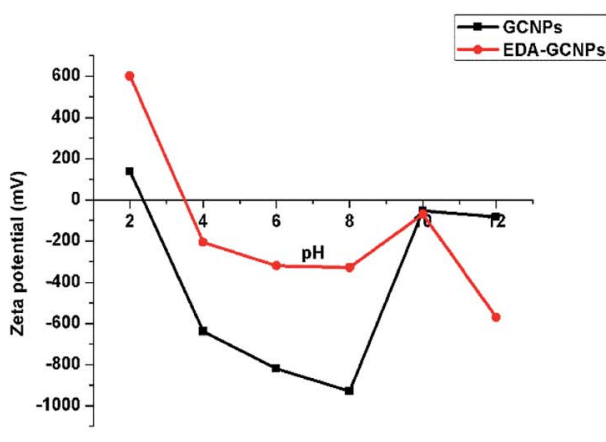

Fig. 2 (a) FTIR spectra, and (b) surface charge analysis results for GCNPs and EDA-GCNPs. 
Table 1 CHNS composition of the GCNPs and EDA-GCNPs

Atomic compositions of elements (atomic \%)

\begin{tabular}{llllll}
\hline & \multicolumn{5}{c}{ Atomic compositions of elements (atomic \%) } \\
\cline { 2 - 6 } Sample & $\mathrm{C}$ & $\mathrm{H}$ & $\mathrm{N}$ & $\mathrm{S}$ & $\mathrm{O}$ \\
\hline GCNPs & 65.88 & 4.22 & - & - & 29.90 \\
EDA-GCNPs & 59.50 & 5.35 & 12.14 & - & 23.90 \\
\hline
\end{tabular}

mesoporous materials (materials with pore diameters ranging from 2-50 nm) and the limiting uptake over a range of high $P /$ $P_{0}$. Another characteristic of this type of isotherm is the slight bend that is observed in low pressures, these characteristics are observed clearly in these materials. An $\mathrm{H} 3$ type hysteresis loop is observed in both isotherms. The hysteresis type is displayed in cases where the isotherm does not exhibit any limiting adsorption at high $P / P_{0}$ and is also normal with aggregates of plate-like particles giving rise to slit-shaped pores. The difference in the sizes of the loops seen in this case might be because of the large difference in surface area. The GCNPs have a higher surface area thus they have a bigger hysteresis loop, this is common in high surface area material. ${ }^{33}$ The introduction of the bulky ethylenediamine groups onto the surface of the GCNPs leads to a drastic decrease in both the surface area from 340.68 to $16 \mathrm{~m}^{2} \mathrm{~g}^{-1}$ and the total pore volume from 0.16 to 0.033 $\mathrm{cm}^{3} \mathrm{~g}^{-1}$. The decrease in surface area and pore size upon modification may be due to amide functional groups being adsorbed and filling the pores of the carbon nanoparticles. ${ }^{21}$

3.1.6. XRD analysis. The XRD patterns of the GCNPs and EDA-GCNPs as presented in Fig. 3c, show that XRD pattern of the EDA functionalized carbon nanoparticles was very similar to that of the unmodified carbon nanoparticles, implying that the functionalization process did not damage the novel structure of the normal CNPs. A broad peak centred at $2 \theta=21.41^{\circ}$ can be seen in both materials and can be attributed to highly disordered carbon atoms that are amorphous in nature as in most carbons and CNPs. The XRD pattern of both GCNPs and EDA-GCNPs showed no additional peaks, thus, we considered the crystal structure of the EDA-GCNPs was the same as that of the normal GCNPs.

\subsection{Adsorption capacity}

3.2.1. Effect of pH. Studies on the effect of pH (Fig. 4a) show that increasing $\mathrm{pH}$ leads to an increase adsorption with a)

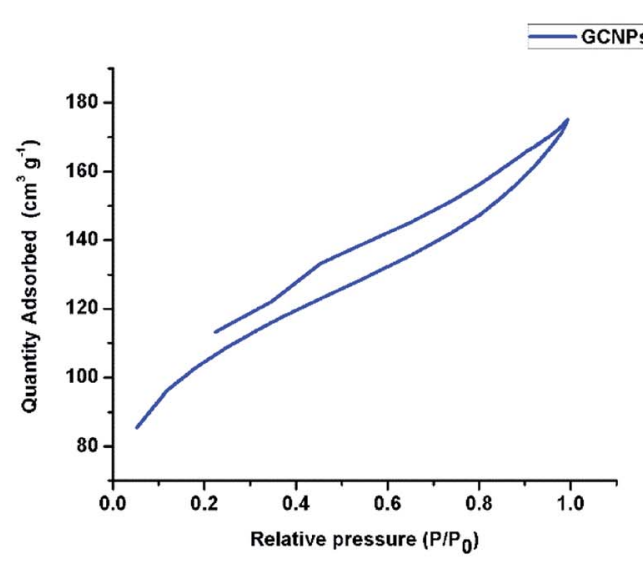

b)
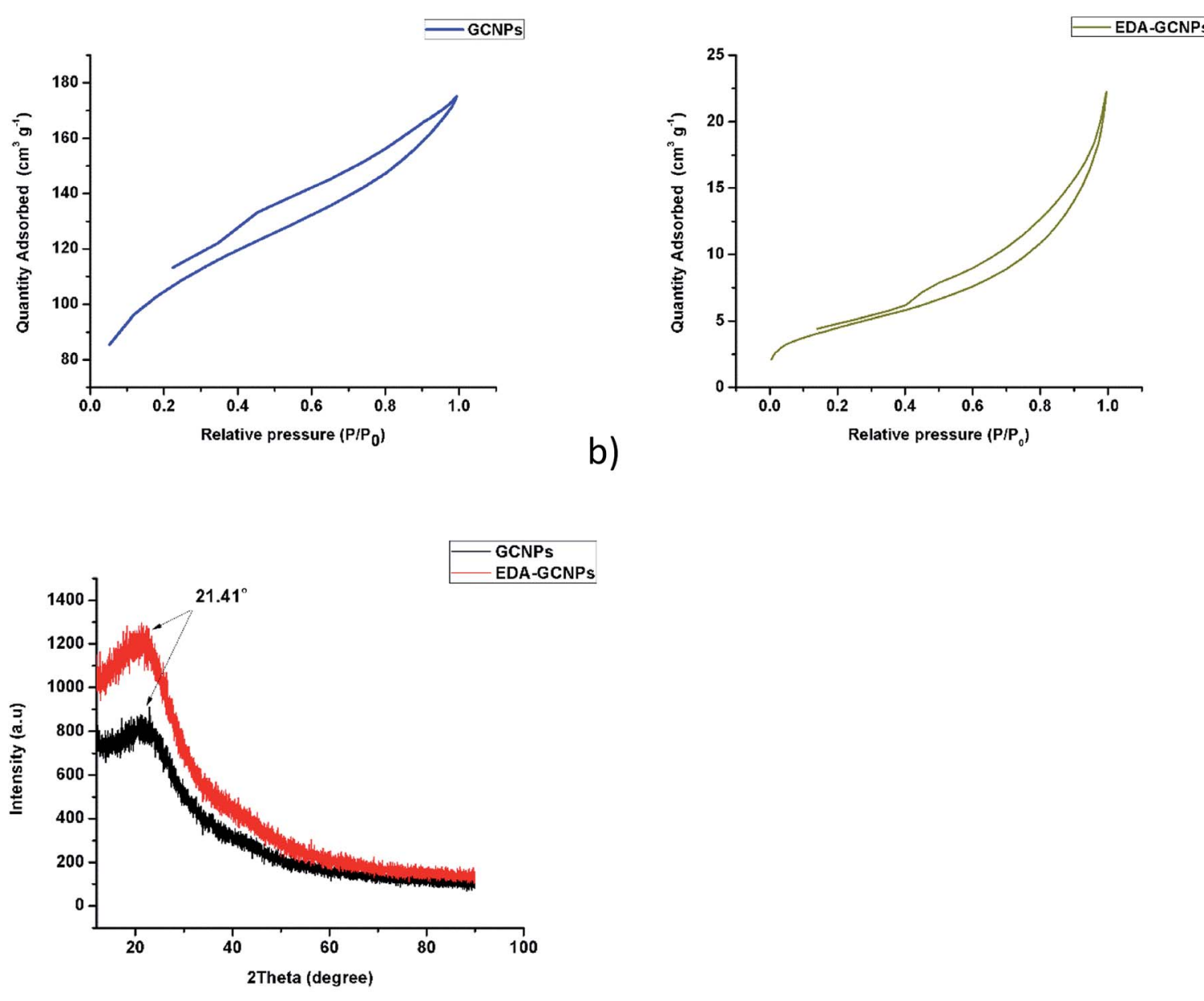

C)

Fig. 3 BET adsorption-desorption isotherms for (a) GCNPs and (b) EDA-GCNPs, as well as (c) XRD diffraction patterns for GCNPs and EDAGCNPs. 
EDA-GCNPs exhibiting a better adsorption capacity. This result strongly agrees with the zeta potential analysis. In a strongly acidic medium (below $\mathrm{pH} 3$ ) the concentration of $\mathrm{H}^{+}$ions on the surface of the adsorbent is high. Therefore, $\mathrm{Cd}^{2+}$ must compete with $\mathrm{H}^{+}$ for active sites on the adsorbent, thus resulting in very little $\mathrm{Cd}^{2+}$ adsorption. The EDA-GCNPs have better adsorption capability because the protonation degree of its amino groups weakens, and the coordination and the chelating ability of these amino groups towards metal ions strengthens resulting in better adsorption. A similar trend was reported by Zhang et al. ${ }^{34}$ Although an increase in $\mathrm{pH}$ resulted in better $\mathrm{Cd}^{2+}$ removal, $\mathrm{pH} 6$ was used in all experiments to avoid the precipitation of cadmium as hydroxides.

3.2.2. Effect of adsorbent dose. After evaluating the effect of mass using 5-100 $\mathrm{mg}$ of the adsorbent, it was concluded that $20 \mathrm{mg}$ was enough to carry out all the adsorption studies, as it gave a higher adsorption capacity at $\mathrm{pH} 6$, in $10 \mathrm{mg} \mathrm{L}^{-1}$ cadmium solution. This was further evidenced by the percentage diagram fitted inside Fig. $4 \mathrm{~b}$, which shows that the $\mathrm{Cd}^{2+}$ removal efficiency increases with increasing adsorbent dose up to the dosage of $20 \mathrm{mg}$, after which the adsorption efficiency remained constant at around 88\% for the GCNPs and around $99 \%$ in the EDA-GCNPs. An increase adsorbent mass means more sites for the adsorbate to bind onto the adsorbent will be available. However, if more of the adsorbent is used with less of the adsorbate $\left(\mathrm{Cd}^{2+}\right)$ present, the adsorption capacity decreases as seen from Fig. $4 \mathrm{~b}$, because there will be nothing to attach to those sites thus a lower adsorption capacity is seen as mass is increased and the volume and concentration of the adsorbent are kept constant.

\subsection{Adsorption kinetics and isotherm analysis}

To study the effect of contact time, $20 \mathrm{mg}$ of the adsorbents and $10 \mathrm{mg} \mathrm{\textrm {L } ^ { - 1 }}$ were agitated at different time intervals as seen in Fig. 5a. Increasing contact time sharply increases the adsorption at a short period of contact time and slowed gradually as equilibrium was approached. This is due to the availability of an initial large number of vacant surface active sites on the two adsorbents. The higher adsorption capacities experienced by the EDA functionalized adsorbent, can still be attributed to the amine functional groups present on its surface, which better chelate to some metal ions better than carboxyl groups contained by the GCNPs. Equilibrium was reached in just $120 \mathrm{~min}$.

The adsorption mechanism of $\mathrm{Cd}^{2+}$ onto the two adsorbents was studied by fitting the linearized pseudo-first-order (eqn (3)) and pseudo-second-order (eqn (4)) kinetic models.

$$
\begin{gathered}
\ln \left(q_{\mathrm{e}}-q_{t}\right)=\ln q_{\mathrm{e}}-k_{1} t \\
\frac{t}{q_{t}}=\frac{1}{k_{2} q_{\mathrm{e}}^{2}}+\frac{1}{q_{\mathrm{e}}} t
\end{gathered}
$$

where $q_{\mathrm{e}}\left(\mathrm{mg} \mathrm{g}^{-1}\right)$ represents the equilibrium adsorption capacity and $q_{t}\left(\mathrm{mg} \mathrm{g}^{-1}\right)$ is the adsorption capacity at a given moment of time, $t$ is the time in minutes, $k_{1}\left(\mathrm{~min}^{-1}\right)$ and $k_{2}(\mathrm{~g}$ $\left.\mathrm{mg}^{-1} \mathrm{~min}^{-1}\right)$ are the pseudo-first order and second-order rate constants, respectively. From eqn (3) and (4), the pseudo-firstorder (Fig. 5b) and pseudo-second-order (Fig. 5c) adsorption model plots were derived. As shown (Table 2), the experimental data best fitted the pseudo-second-order model, as indicated by the higher correlation coefficients $\left(R^{2}=0.9967\right.$ and 0.9994 , for the GCNPs and EDA-GCNP, respectively). Based on the pseudosecond-order model, the adsorption of Cd onto the two adsorbents is largely influenced by the functional groups present, hence chemisorption is the driving mechanism in the adsorption processes as deduced from the kinetics data.

To understand the adsorbate-adsorbent interactions (which are vital when designing adsorption systems), and to obtain the maximum adsorption capacity for EDA-GCNPs, the linearized Langmuir (eqn (5)) and Freundlich (eqn (6)) equations were used.

$$
\begin{gathered}
\frac{C_{\mathrm{e}}}{q_{\mathrm{e}}}=\frac{1}{q_{\mathrm{m}} b}+\frac{C_{\mathrm{e}}}{q_{\mathrm{m}}} \\
\ln q_{\mathrm{e}}=\ln K_{\mathrm{F}}+\frac{1}{n} \ln C_{\mathrm{e}}
\end{gathered}
$$

where $C_{\mathrm{e}}\left(\mathrm{mg} \mathrm{L}^{-1}\right)$ is the final concentration. $q_{\mathrm{m}}$ is the saturated adsorption capacity $\left(\mathrm{mg} \mathrm{g}^{-1}\right), b$ is adsorption constant $\left(\mathrm{L} \mathrm{mg}^{-1}\right)$,

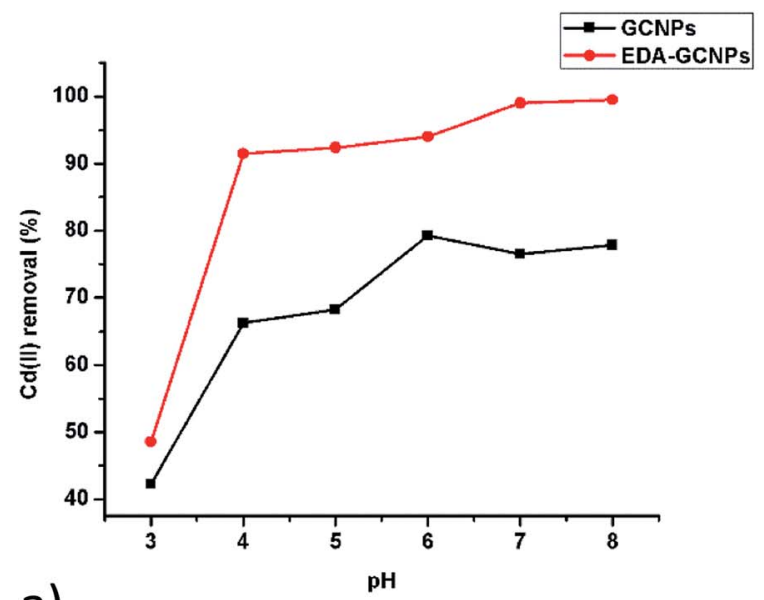

a)

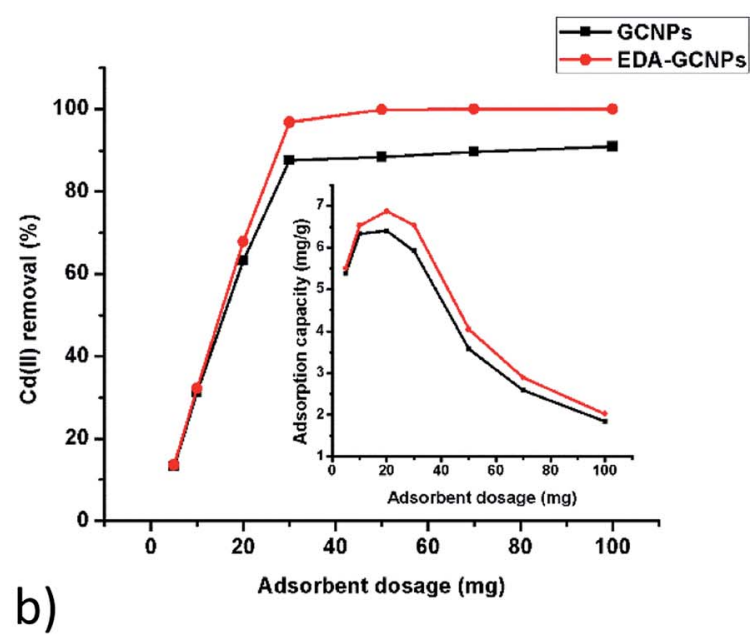

Fig. 4 Effect of (a) $\mathrm{pH}$ and (b) adsorbent dosage on the adsorption of $\mathrm{Cd}(॥)$ by GCNPs and EDA-GCNPs. 


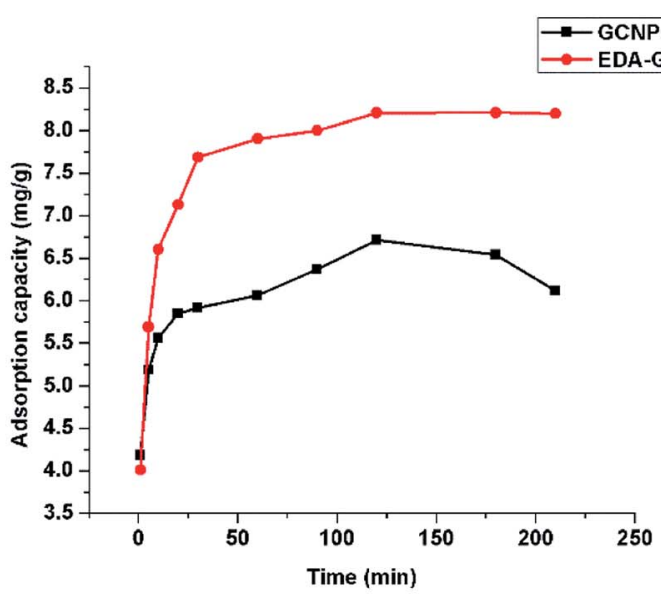

a)

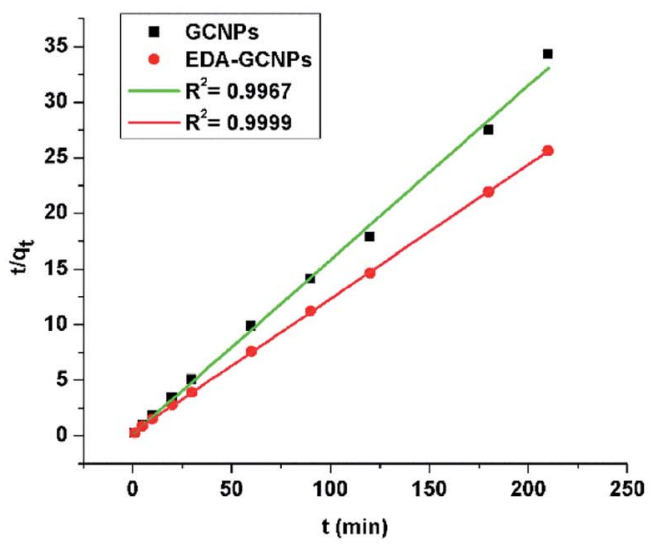

c)

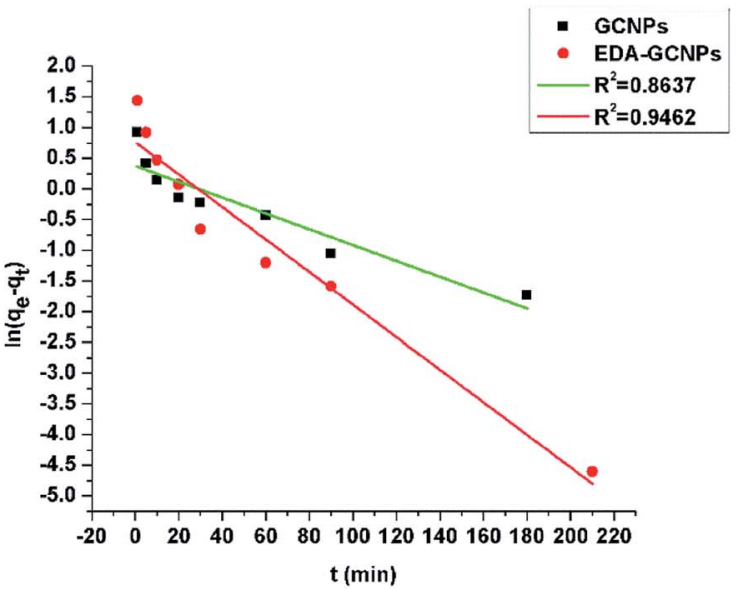

b)

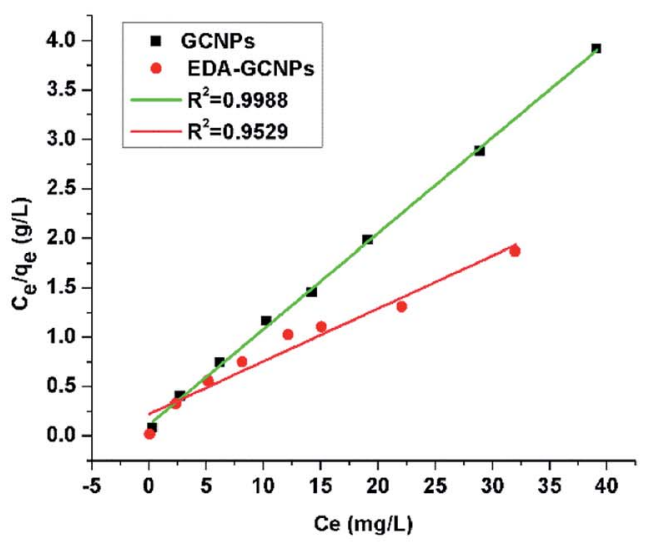

d)

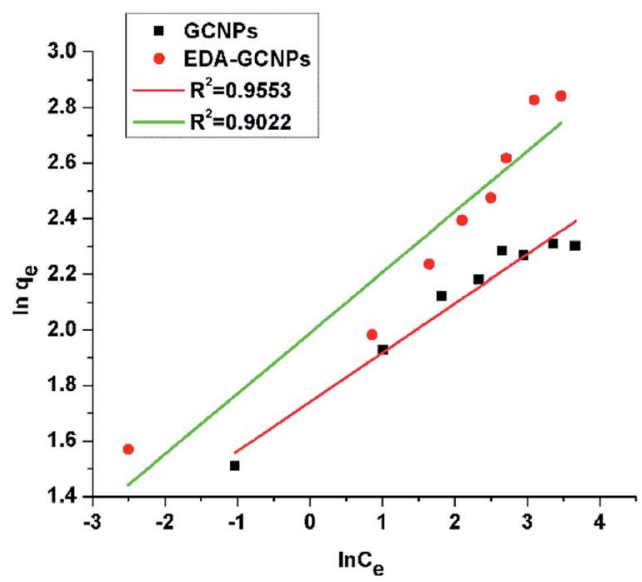

e)

Fig. 5 Rate and equilibrium studies; (a) effect of contact time, linearized (b) pseudo-first-order, and (c) pseudo-second-order kinetic models, (d) Langmuir, and (e) Freundlich adsorption isotherms.

$K_{\mathrm{F}}\left(\mathrm{mg} \mathrm{g}^{-1}\right)$ and $n$ are Freundlich constants. The Langmuir plots depicted in Fig. 5d, was linear in the concentration range of 5$50 \mathrm{mg} \mathrm{L}^{-1}$ with higher correlation coefficients (Table 3) when compared to the Freundlich adsorption model (Fig. 5e), implying that the Langmuir adsorption model best describes the adsorption data and a monolayer adsorption occurred. The 
Table 2 Parameters for pseudo-first-order and pseudo-second-order kinetic models

\begin{tabular}{|c|c|c|c|c|c|c|c|}
\hline Adsorbent & $\frac{\text { Experimental }}{q_{\mathrm{e}}}$ & \multicolumn{3}{|c|}{ Pseudo-first-order } & \multicolumn{3}{|c|}{ Pseudo-second-order } \\
\hline EDA-GCNPs & 8.207 & 2.155 & 0.0265 & 0.9462 & 8.301 & 0.0502 & 0.9999 \\
\hline
\end{tabular}

EDA-GCNPs (18.707 $\mathrm{mg} \mathrm{g}^{-1}$ ) had a greater adsorption capacity than the GCNPs $\left(10.3135 \mathrm{mg} \mathrm{g}^{-1}\right)$, meaning the functionalization greatly improved the adsorption of $\mathrm{Cd}^{2+}$ onto the carbon nanoparticles.

3.3.1. Comparison of cadmium removal onto GCNPs and EDA-GCNPs. The removal efficiencies and kinetics of $\mathrm{Cd}^{2+}$ onto GCNPs and EDA-GCNPs were compared using several parameters such as the initial $\mathrm{Cd}^{2+}$ concentration of $5-50 \mathrm{mg} \mathrm{L}^{-1}$, $20 \mathrm{mg}$ adsorbent and $\mathrm{pH}$ of 6 , at $25^{\circ} \mathrm{C}$ as well as varying time from 5-220 min. The results are shown in Tables 2 and 3, from these results it can be inferred that modifying the GCNPs with EDA greatly enhances the removal capacity and that the functional groups introduced onto the surface of the carbon nanoparticles were behind the adsorption process (chemisorption).

3.3.2. Comparison of cadmium adsorption against various adsorbents. Table 3 lists the values of the maximum adsorption capacities for $\mathrm{Cd}^{2+}$ adsorption on different adsorbents which included the adsorbent of this work and several adsorbents reported in literature. Due to the differences in experimental conditions, a direct comparison between the EDA-GCNPs and the other adsorbents is difficult. However, it can be found that the maximum adsorption capacity of the modified material was high as compared with most adsorbents presented in Table 4.

\subsection{Thermodynamics}

The adsorption parameters such as standard Gibbs free energy $\left(\Delta G^{\circ}\right)$, enthalpy change $\left(\Delta H^{\circ}\right)$ and entropy change $\left(\Delta S^{\circ}\right)$ were calculated using the data obtained from the effect of temperature through the Van't Hoff equations.

$$
\begin{aligned}
& \ln K=\frac{\Delta S^{\circ}}{R}-\frac{\Delta H^{\circ}}{R T} \\
& \Delta G=-R T \ln K
\end{aligned}
$$

The value of $K$ was obtained from the ratio of adsorbate particles attached to the surface of the adsorbent to that of the adsorbate particles in the aqueous solution. $R$ is gas constant

Table 3 Parameters for the Langmuir and Freundlich adsorption models

\begin{tabular}{lllllllll}
\hline & \multicolumn{2}{l}{ Langmuir model } & & & \multicolumn{2}{c}{ Freundlich model } \\
\cline { 2 - 3 } Adsorbent & $q_{\mathrm{m}}$ & $B$ & $R^{2}$ & & $n$ & $K_{\mathrm{F}}$ & $R^{2}$ \\
\hline GCNPs & 10.314 & 0.884 & 0.9988 & & 5.6 & 1.74 & 0.9553 \\
EDA-GCNPs & 18.708 & 0.0265 & 0.9529 & & 4.6 & 1.24 & 0.9022
\end{tabular}

(8.314 $\mathrm{J} \mathrm{K}^{-1} \mathrm{~mol}^{-1}$ ), $T$ is the temperature (kelvin). Eqn (7), is a plot of $\ln K v s .1 / T$, and yields a straight line from which $\Delta S^{\circ}$ and $\Delta H^{\circ}$ can be calculated from the intercept and slope respectively. ${ }^{40}$ The plot of $\ln K v s .1 / T$ for the GCNPs and EDAGCNPs is expressed in Fig. 6, and the parameters are tabled as follows (Table 5).

The positive nature of the $\Delta H^{\circ}$ values for the two materials implies that the adsorption process is endothermic as adsorption of the $\mathrm{Cd}^{2+}$ increased upon an increment of temperature. The higher magnitude of $\Delta H^{\circ}$ may support the earlier argument that the chelation (a chemical bonding) play a role in the adsorption process. This bonding may be the reason for the increase in enthalpy. A positive value $\Delta S^{\circ}$ value shows the enhanced randomness at the adsorbent/solution interface during the $\mathrm{Cd}^{2+}$ adsorption on the active sites of the adsorbent,

Table 4 Adsorption capacities for $\mathrm{Cd}^{2+}$ onto EDA-GCNPs and various

\begin{tabular}{lcl}
\hline & $\begin{array}{l}\text { Adsorption capacity } \\
\left(\mathrm{mg} \mathrm{g}^{-1}\right)\end{array}$ & Reference \\
\hline Odsorbent & 10.86 & 35 \\
Activated carbon & 4.29 & 36 and 37 \\
Cystine modified biomass & 11.63 & 38 \\
Cellu/cys-bent & 18.52 & 39 \\
nanocomposite & & This work \\
EDA-GCNPS & 18.71 &
\end{tabular}

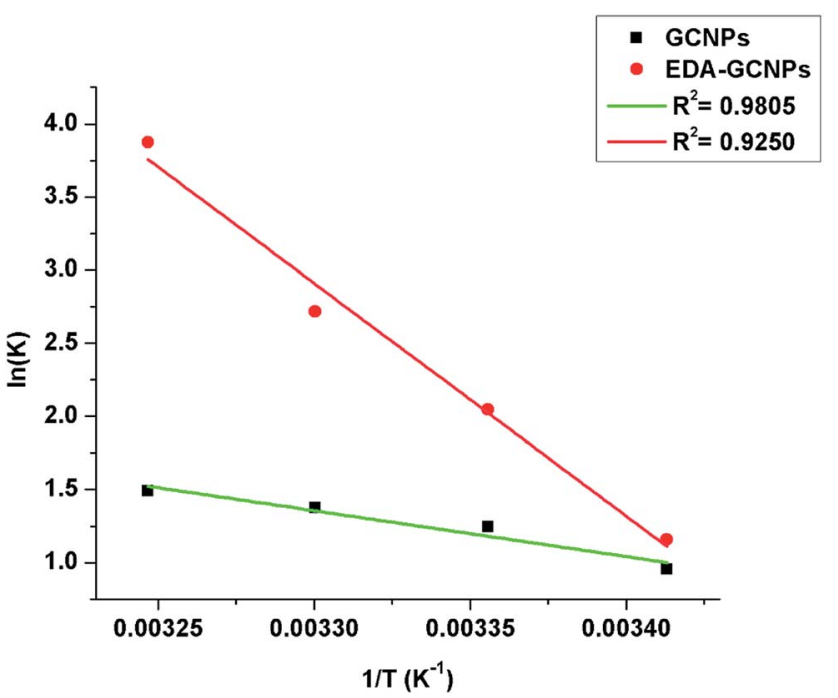

Fig. 6 Van't Hoff linear plot of $\ln K$ versus $1 / T$ for $\mathrm{Cd}^{2+}$ on GCNPs and EDA-GCNPs. 
Table 5 Thermodynamic parameters of adsorbents for adsorption of $\mathrm{Cd}^{2+a}$

\begin{tabular}{llll}
\hline Adsorbent & $\Delta H^{\circ}$ & $\Delta S^{\circ}$ & $\Delta G^{\circ}$ \\
\hline GCNPs & 26.11 & 0.097 & $-2.32 @ 293 \mathrm{~K}$ \\
& & & $-3.08 @ 298 \mathrm{~K}$ \\
& & $-3.46 @ 303 \mathrm{~K}$ \\
EDA-GCNPs & \multirow{2}{*}{132.29} & 0.46 & $-2.81 @ 308 \mathrm{~K}$ \\
& & & $-5.07 @ 293 \mathrm{~K}$ \\
& & & $-6.85 @ 303 \mathrm{~K}$ \\
& & & $-9.92 @ 308 \mathrm{~K}$
\end{tabular}

${ }^{a} \Delta H^{\circ} \& \Delta G^{\circ}=\mathrm{kJ} \mathrm{mol}^{-1}, \Delta S^{\circ}=\mathrm{kJ} \mathrm{mol}^{-1} \mathrm{~K}^{-1}$.

and finally, the negative $\Delta G^{\circ}$ values obtained at various temperatures portray the spontaneous nature of the adsorption process when the two materials are applied.

\section{Conclusion}

Carbon nanoparticles were successfully synthesised from glucose and modified with ethylenediamine, using HATU as the coupling agent. The characterization after ethylenediamine confirmed the amidation step which enhanced the adsorption process. The carbon nanoparticles synthesised in this manner show promise in the field of adsorption, as they are cheap to make, reusable and easy to functionalize, thus creating possibilities in the removal of many pollutants (both organic and inorganic).

\section{Conflict of interest}

There are no conflicts of interest to declare.

\section{Acknowledgements}

Financial supports from the following institutions in South Africa are gratefully acknowledged: DST/Mintek Nanotechnology Innovation Centre, University of Johannesburg; Centre for Nanomaterials Science Research, University of Johannesburg; National Research Foundation of South Africa (CPRR Grant Number: 98887).

\section{References}

$1 \mathrm{~S}$. Oelofse and W. Strydom, A CSIR perspective on water in South Africa 2010, retrieved from, http://www.theeis.com/ data/literature/CSIR\%20Perspective\%20on\%20Water\% 20in\%20South\%20Africa\%202010.pdf, 24 June 2017.

2 E. Repo, J. K. Warchoł, A. Bhatnagar, A. Mudhoo and M. Sillanpää, Water Res., 2013, 47, 4812-4832.

3 D. Sounthararajah, P. Loganathan, J. Kandasamy and S. Vigneswaran, J. Hazard. Mater., 2015, 287, 306-316.

4 A. Bernard, Indian J. Med. Res., 2008, 128, 557.

5 Y. Wang, G. Ye, H. Chen, X. Hu, Z. Niu and S. Ma, J. Mater. Chem. A, 2015, 3, 15292-15298.

6 L. Charerntanyarak, Water Sci. Technol., 1999, 39, 135-138.
7 E. Saljoughi and S. M. Mousavi, Sep. Purif. Technol., 2012, 90, 22-30.

8 S. Ahmed, S. Chughtai and M. A. Keane, Sep. Purif. Technol., 1998, 13, 57-64.

9 A. H. Mahvi and E. Bazrafshan, World Appl. Sci. J., 2007, 2, 34-39.

10 C.-P. Huang and F. Ostovic, J. Environ. Eng. Div., 1978, 104, 863-878.

11 Y.-H. Li, S. Wang, Z. Luan, J. Ding, C. Xu and D. Wu, Carbon, 2003, 41, 1057-1062.

12 Ş. Parlayıcı and E. Pehlivan, Powder Technol., 2017, 317, 2330.

13 R. Sharma, A. Sarswat, C. U. Pittman and D. Mohan, RSC Adv., 2017, 7, 8606-8624.

14 W.-K. Kim, T. Shim, Y.-S. Kim, S. Hyun, C. Ryu, Y.-K. Park and J. Jung, Bioresour. Technol., 2013, 138, 266-270.

15 H. Liu, T. Ye and C. Mao, Angew. Chem., Int. Ed., 2007, 46, 6473-6475.

16 H. Zhu, X. Wang, Y. Li, Z. Wang, F. Yang and X. Yang, Chem. Commun., 2009, 5118-5120.

17 H. Li, X. He, Y. Liu, H. Huang, S. Lian, S.-T. Lee and Z. Kang, Carbon, 2011, 49, 605-609.

18 Z. Jiang, J. Jin, C. Xiao and X. Li, Colloids Surf., A, 2012, 395, 105-115.

19 Q. Li, N. Yu, Z. Qiu, X. Zhou and C. F. Wu, Colloids Surf., A, 2008, 317, 87-92.

20 P. Jal, S. Patel and B. Mishra, Talanta, 2004, 62, 1005-1028. 21 S. Trostová, I. Stibor, J. Karpíšková, Z. Kolská and V. Švorčík, Mater. Lett., 2013, 102, 83-86.

22 C. Wang, X. Zhang, F. Lv and L. Peng, J. Appl. Polym. Sci., 2012, 124, 5194-5199.

23 P. Scharff, Carbon, 1998, 36, 481-486.

24 S. Iijima and T. Ichihashi, Nature, 1993, 363, 603-605.

25 D. Mohan and C. U. Pittman, J. Hazard. Mater., 2006, 137, 762-811.

26 D. Sud, G. Mahajan and M. Kaur, Bioresour. Technol., 2008, 99, 6017-6027.

27 J. P. Ruparelia, S. P. Duttagupta, A. K. Chatterjee and S. Mukherji, Desalination, 2008, 232, 145-156.

28 S. Chandra, P. Das, S. Bag, D. Laha and P. Pramanik, Nanoscale, 2011, 3, 1533-1540.

29 J. Shen, W. Huang, L. Wu, Y. Hu and M. Ye, Mater. Sci. Eng., A, 2007, 464, 151-156.

30 J. L. Stevens, A. Y. Huang, H. Peng, I. W. Chiang, V. N. Khabashesku and J. L. Margrave, Nano Lett., 2003, 3, 331-336.

31 H. Peng, L. B. Alemany, J. L. Margrave and V. N. Khabashesku, J. Am. Chem. Soc., 2003, 125, 1517415182.

32 J.-l. Yan, G.-j. Chen, C. Jun, Y. Wei, B.-h. Xie and M.-b. Yang, New Carbon Mater., 2012, 27, 370-376.

33 M. X. Pulikkathara, O. V. Kuznetsov and V. N. Khabashesku, Chem. Mater., 2008, 20, 2685-2695.

34 F. Zhang, B. Wang, S. He and R. Man, J. Chem. Eng. Data, 2014, 59, 1719-1726.

35 Y.-H. Li, J. Ding, Z. Luan, Z. Di, Y. Zhu, C. Xu, D. Wu and B. Wei, Carbon, 2003, 41, 2787-2792. 
36 N. Kannan and G. Rengasamy, Water, Air, Soil Pollut., 2005, 163, 185-201.

37 J. Goel, K. Kadirvelu, C. Rajagopal and V. K. Garg, J. Hazard. Mater., 2005, 125, 211-220.

38 J. Yu, M. Tong, X. Sun and B. Li, J. Hazard. Mater., 2007, 143, 277-284.
39 R. Ahmad and I. Hasan, Sep. Sci. Technol., 2016, 51, 381-394. 40 H. Wang, Y.-g. Liu, G.-m. Zeng, X.-j. Hu, X. Hu, T.-t. Li, H.-y. Li, Y.-q. Wang and L.-h. Jiang, Carbohydr. Polym., 2014, 113, 166-173. 